\title{
Safety and efficacy of ospemifene for the treatment of dyspareunia associated with vulvar and vaginal atrophy due to menopause
}

REVIEW

\section{Gregory T Wurz \\ Chiao-Jung Kao \\ Michael W DeGregorio}

Department of Internal Medicine, Division of Hematology and Oncology, University of California Davis, Sacramento, CA, USA

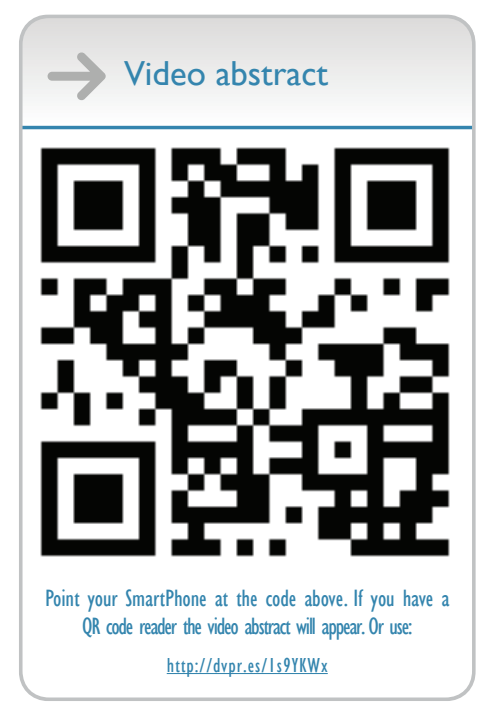

Correspondence: Michael W DeGregorio Department of Internal Medicine, Division of Hematology and Oncology, University of California Davis, $4501 \times$ Street, Suite 3016, Sacramento, CA 95817, USA

$\mathrm{Tel}+\mathrm{l} 9167342360$

Fax + I 9167342070

Email mwdegregorio@ucdavis.edu
This article was published in the following Dove Press journal:

Clinical Interventions in Aging

13 November 2014

Number of times this article has been viewed

Abstract: During the menopausal transition, women experience a number of symptoms due to declining estrogen levels, including vasomotor symptoms and vulvar and vaginal atrophy (VVA). Unlike vasomotor symptoms, vaginal dryness and dyspareunia, the main symptoms of VVA, typically worsen without treatment and can significantly impact the quality of life. Up to $60 \%$ of postmenopausal women may be affected by VVA, but many women unfortunately do not seek treatment due to embarrassment or other factors. After 20+ years in development, ospemifene (Osphena ${ }^{\mathrm{TM}}$ ) was approved by the US Food and Drug Administration in 2013 for treatment of moderate-to-severe dyspareunia associated with VVA due to menopause. As the first non-hormonal alternative to estrogen-based products for this indication, the approval of ospemifene represents a significant milestone in postmenopausal women's health. Ospemifene is a non-steroidal estrogen receptor agonist/antagonist, also known as a selective estrogen receptor modulator (SERM), from the same chemical class as the breast cancer drugs tamoxifen and toremifene. Unlike other selective estrogen receptor modulators, ospemifene exerts a strong, nearly full estrogen agonist effect in the vaginal epithelium, making it well suited for the treatment of dyspareunia in postmenopausal women. Results of Phase III clinical trials showed that ospemifene significantly improved the vaginal maturation index (decreased parabasal cells and increased superficial cells), decreased vaginal $\mathrm{pH}$, and decreased severity of the self-identified most bothersome symptom (dyspareunia or vaginal dryness) compared to placebo. Long-term safety studies revealed that $60 \mathrm{mg}$ ospemifene given daily for 52 weeks was well tolerated and was not associated with any endometrium or breast-related safety concerns. This review discusses the preclinical and clinical data supporting the use of ospemifene for the treatment of dyspareunia associated with VVA due to menopause and provides an overview of its clinical safety.

Keywords: genitourinary syndrome of menopause, SERM, sexual dysfunction

\section{Introduction}

Ospemifene is an estrogen receptor agonist/antagonist, also known as a selective estrogen receptor modulator (SERM), from the same chemical class (triphenylethylenes) as tamoxifen and toremifene, both of which are used in the treatment of breast cancer. Ospemifene is, in fact, one of the major metabolites of toremifene. After over 20 years in development, ospemifene was approved in early 2013 by the US Food and Drug Administration (FDA) for the treatment of moderate-to-severe dyspareunia associated with vulvar and vaginal atrophy (VVA) due to menopause. The latest terminology endorsed by the North American Menopause Society and the International Society for the Study of Women's Sexual Health replaces use of the term VVA with genitourinary syndrome of menopause (GSM). As the first non-hormonal treatment for dyspareunia, the approval of ospemifene, sold under the trade name Osphena ${ }^{\mathrm{TM}}$, 
represents a significant milestone in postmenopausal women's health. The complete developmental history of ospemifene is discussed in a recently published book ${ }^{1}$ as well as a recent review article in the journal Steroids. ${ }^{2}$ Prior to the approval of ospemifene, the only FDA-approved treatments for dyspareunia contained estrogen, which when taken orally has been associated with an increased risk of breast cancer ${ }^{3}$ and other potentially serious complications ${ }^{4-6}$ in large clinical trials of hormone replacement therapy. Figure 1 shows the chemical structures of ospemifene and its known metabolites that have been detected in vivo. ${ }^{7}$

The precipitous decline in the use of estrogen-based therapies for menopausal symptoms following the results of the Women's Health Initiative trials of estrogen plus progestin ${ }^{5}$ and estrogen alone ${ }^{8}$ created an unmet medical need for an effective, non-hormonal alternative to estrogen for treating the symptoms of VVA. While over-the-counter moisturizers and lubricants can bring temporary relief and are recommended for postmenopausal women experiencing mild symptoms, ${ }^{9}$ they do not treat the underlying condition and are generally ineffective for women experiencing moderate-to-severe symptoms. ${ }^{10}$ Uniquely, among the FDA-approved SERMs tamoxifen, toremifene, raloxifene, and bazedoxifene, which is approved as a single agent only in Europe, ospemifene exerts a strong, beneficial estrogen agonist effect on the vaginal epithelium, a property that was first observed in Phase I clinical trials. ${ }^{11}$ The recognition of ospemifene's strong estrogenic effects in the vagina ultimately led to its development for the treatment of dyspareunia rather than postmenopausal osteoporosis or breast cancer chemoprevention, which were the originally targeted indications.

Dyspareunia, or painful sexual intercourse, along with vaginal dryness, itching and incontinence, are the major symptoms of VVA, also known as atrophic vaginitis, which is a common condition in postmenopausal women characterized by thinning of the vaginal epithelium and atrophy of the vulva, vagina, and urinary tract. ${ }^{12}$ The symptoms of VVA are chronic and progressive without treatment in most postmenopausal women, eventually leading to the deterioration of urogenital health and sexual dysfunction, ${ }^{13}$ which can in turn adversely affect postmenopausal women physically, psychologically, and socially. ${ }^{14}$ Approximately $60 \%$ of postmenopausal women who have never been treated with hormone therapy suffer from $\mathrm{VVA}^{12,15,16}$ and $>30 \%$ of women who experience dyspareunia and/or vaginal dryness do not report their symptoms to their physicians. ${ }^{12,13,15,17}$ These symptoms are a result of the declining estrogen level seen in perimenopause and postmenopause, which<smiles>OC=COc1ccc(C(=C(CCCl)c2ccccc2)c2ccc(O)cc2)cc1</smiles>

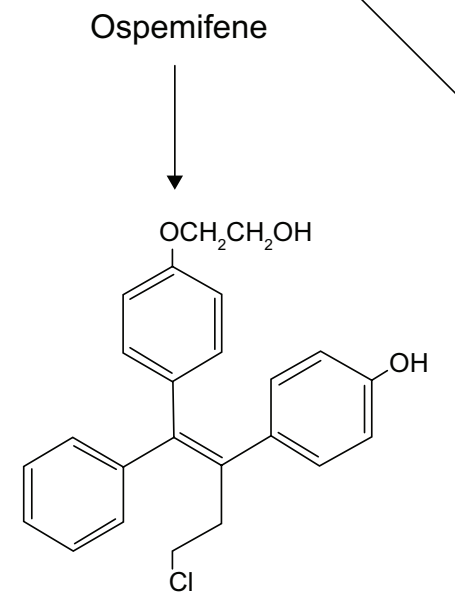

4'-hydroxyospemifene (5\%)

4-hydroxyospemifene (25\%)

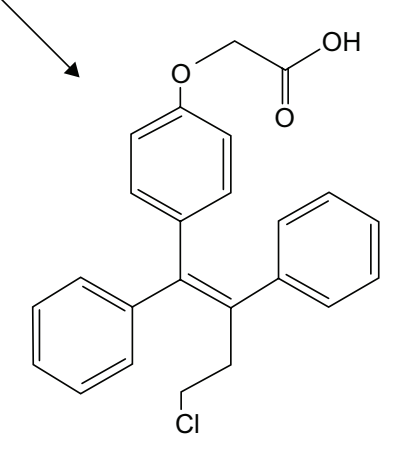

Carboxylic acid (1\%-2\%)

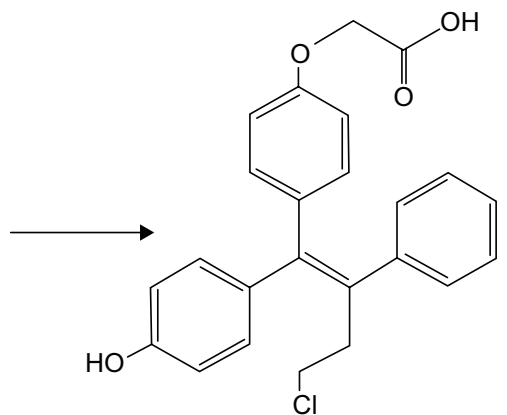

4-hydroxycarboxylic acid (1\%-2\%)

Figure I Metabolism of ospemifene in humans following a single oral dose of $\left[{ }^{3} \mathrm{H}\right]$-ospemifene. 
leads to changes in the cellular composition of the vaginal epithelium as well as vaginal $\mathrm{pH} .{ }^{10}$ One popular measure of VVA is what is known as the vaginal maturation index. The vaginal maturation index measures the ratios of the different cell types in the vaginal epithelium. Increases in superficial and intermediate cells and a decrease in parabasal cells indicate an improvement in VVA. Vaginal $\mathrm{pH}$ is normally acidic in premenopausal women, which helps keep microbial growth in check, thereby preventing infection. During the menopausal transition, $\mathrm{pH}$ levels begin to increase, which can then lead to an increase in yeast and urinary tract infections. ${ }^{18}$ Effective treatment of VVA, as with ospemifene or an estrogen-based therapy, leads to a decrease in vaginal $\mathrm{pH}$. Thinning of the vaginal wall, reduced natural lubrication due to vaginal dryness, reduced tissue elasticity, and a shortening and narrowing of the vagina can lead to dyspareunia. ${ }^{19}$

With respect to the safety and efficacy of ospemifene in the treatment of dyspareunia associated with VVA due to menopause, this review discusses: (1) the preclinical effects of ospemifene in the vagina in the ovariectomized rat model of menopause; (2) the efficacy of ospemifene in treating VVA due to menopause in clinical trials as assessed by improvement in the vaginal maturation index, decrease in vaginal $\mathrm{pH}$, and improvement in self-identified most bothersome symptom; and (3) the overall safety profile of ospemifene in clinical trials.

\section{Preclinical studies}

The effects of ospemifene on vaginal weight and epithelial height were examined in three studies utilizing the ovariectomized rat model of menopause. ${ }^{20,21}$ In the first study, groups of rats were treated with three different doses $(10 \mathrm{mg} / \mathrm{kg}, 30 \mathrm{mg} / \mathrm{kg}$, and $100 \mathrm{mg} / \mathrm{kg}$ orally) of ospemifene daily for 2-4 weeks and compared to rats treated with raloxifene $10 \mathrm{mg} / \mathrm{kg}$, 17- $\alpha$-ethinyl estradiol $\left(\mathrm{EE}_{2}\right) 100 \mu \mathrm{g} / \mathrm{kg}$, or control (no treatment). When compared to the control rats, all three doses of ospemifene at both two and four weeks of treatment resulted in significantly increased vaginal weight and epithelial height that did not significantly differ from rats treated with $\mathrm{EE}_{2}$, which was suggestive of a full estrogen agonist effect. The effects of ospemifene on vaginal epithelial height were maintained up to 2 weeks after cessation of dosing. ${ }^{21}$ In contrast, raloxifene produced increases in vaginal weight and epithelial height only one-half and one-tenth that of $\mathrm{EE}_{2}$, respectively. ${ }^{20}$ This result was not unexpected because raloxifene has not been shown to have any meaningful effects on the vaginal epithelium. ${ }^{22}$ In agreement with earlier studies, ${ }^{23}$ ospemifene was found to have a partial estrogen agonist effect on uterine weight, similar to raloxifene. ${ }^{21}$

Utilizing the same ovariectomized rat model of menopause, the dose response effects of both ospemifene and its main metabolite 4-hydroxyospemifene on vaginal weight and epithelial height were evaluated and compared to the synthetic estrogen $\mathrm{EE}_{2}$ in two additional experiments. 4-Hydroxyospemifene is by far the most common metabolite produced in vivo, followed by $4^{\prime}$-hydroxyospemifene and the carboxylated metabolites, which represent only $1 \%-2 \%$ of total ospemifene (Figure 1). ${ }^{7}$ Different groups of rats were given a range of doses of ospemifene $(0.01 \mathrm{mg} / \mathrm{kg}, 0.1 \mathrm{mg} / \mathrm{kg}$, $0.3 \mathrm{mg} / \mathrm{kg}, 1 \mathrm{mg} / \mathrm{kg}, 3 \mathrm{mg} / \mathrm{kg}$, and $10 \mathrm{mg} / \mathrm{kg}$ ) and its metabolite $(0.01 \mathrm{mg} / \mathrm{kg}, 0.1 \mathrm{mg} / \mathrm{kg}, 0.3 \mathrm{mg} / \mathrm{kg}$, and $1 \mathrm{mg} / \mathrm{kg})$ daily for 2 weeks compared to a fixed dose of $\mathrm{EE}_{2}(100 \mu \mathrm{g} / \mathrm{kg})$, all of which were administered orally. With respect to vaginal epithelial height, the half maximal effective doses $\left(\mathrm{ED}_{50}\right)$ of ospemifene and 4-hydroxyospemifene were found to be $0.39 \mathrm{mg} / \mathrm{kg}$ and $0.28 \mathrm{mg} / \mathrm{kg}$, respectively, while the calculated $\mathrm{ED}_{50}$ values for vaginal weight were $0.48 \mathrm{mg} / \mathrm{kg}$ and $4.6 \mathrm{mg} / \mathrm{kg}$, respectively, for ospemifene and its metabolite. A clear dose response effect of ospemifene was observed, with all doses but the lowest dose producing a significant increase in vaginal epithelial height, and the three highest doses causing significant increases in vaginal weight compared to ovariectomized control rats. Only the highest dose of 4-hydroxyospemifene produced a significant increase in vaginal weight. The maximal effect of ospemifene on vaginal epithelial height was nearly equivalent to $\mathrm{EE}_{2}$, again suggesting a full estrogen agonist effect, while the effect of the metabolite was suggestive of a partial agonist. Histological evaluation revealed that ospemifene treatment resulted in increased thickness, mucification, and vacuolization of the vaginal luminal epithelium, ${ }^{21}$ while missing vacuoles and only minor increases in mucification were observed with raloxifene treatment. ${ }^{20}$

Two additional studies were performed to specifically evaluate the effects of ospemifene on vaginal histology in the ovariectomized rat model of menopause. ${ }^{21}$ The first of these was a pilot study in which ospemifene was given orally or by vaginal suppository daily for 14 days at a dose of $1 \mathrm{mg} / \mathrm{kg}$ and compared to orally administered $\mathrm{EE}_{2}$ (100 $\mu \mathrm{g} / \mathrm{kg})$. Ospemifene, whether given orally or by suppository, increased the thickness of the vaginal epithelium and increased expression of the progesterone receptor (PR) in the epithelium as well as the underlying elastic (lamina propria) and muscular (lamina muscularis) tissue layers of the vagina in a manner similar to $\mathrm{EE}_{2}$. To confirm the results 
of the pilot study, a second study with the same design was conducted examining the effects of ospemifene compared to $\mathrm{EE}_{2}$ on the vaginal epithelium and $\mathrm{PR}$ expression. Again, regardless of the route of drug administration, orally or locally, the effects of ospemifene on the thickness of the vaginal epithelium and PR expression in the epithelium and underlying tissue layers were virtually equivalent to $\mathrm{EE}_{2}$, confirming a full estrogen agonist effect. The only observed difference between ospemifene and $\mathrm{EE}_{2}$ was with respect to cornification of the vaginal epithelium, which was absent in the ospemifene-treated rats. ${ }^{21}$

\section{Clinical studies}

\section{Phase I}

Two early Phase I clinical trials of ospemifene were conducted to evaluate the safety, tolerability, pharmacokinetics, and pharmacodynamics of ospemifene following single oral doses in male volunteers and repeated daily oral dosing for 12 weeks in healthy postmenopausal women. ${ }^{11,24}$ The latter trial was a randomized, placebo-controlled, double-blind study performed in 40 healthy postmenopausal women in order to define a safe and effective dose range for Phase II studies. ${ }^{11}$ Women in this study were randomized to receive daily doses of placebo or ospemifene at doses of $25 \mathrm{mg}$, $50 \mathrm{mg}, 100 \mathrm{mg}$, or $200 \mathrm{mg}$ for 12 weeks. At each dose level, a total of ten subjects were randomly assigned to receive placebo (two subjects) or ospemifene (eight subjects). In addition to standard laboratory and adverse event monitoring, the following parameters were evaluated: (1) estrogenresponsive hormonal markers, (2) estrogen-related changes in the endometrium, (3) changes in the vaginal maturation index (parabasal, intermediate, and superficial cells of the vaginal epithelium), and (4) clinical evaluations of vasomotor symptoms.

The results of this study showed that ospemifene was well tolerated and caused no hematologic, hepatic, or renal abnormalities. At doses higher than $100 \mathrm{mg}$ /day, ospemifene caused significant increases in serum follicle-stimulating hormone, which is an indicator of estrogenic pituitary feedback, but no changes in serum estradiol or luteinizing hormone were observed. With respect to the vaginal maturation index, all study subjects had an atrophic vaginal epithelium at baseline. All doses of ospemifene exerted a clear estrogenic effect on the vaginal epithelium as shown by significant decreases in the percentages of parabasal cells and significant increases in the percentages of superficial cells compared to placebo. Regarding endometrial safety, no clinically significant changes in endometrial thickness as assessed by transvaginal ultrasound were observed at any ospemifene dose level, and only minimal proliferative changes were seen mostly at the $100 \mathrm{mg}$ and $200 \mathrm{mg}$ doses. No incidences of endometrial hyperplasia or bleeding were observed at any dose. Although no significant changes in menopausal symptoms were observed, there was a tendency for these symptoms to decrease at the lower doses and increase at the higher doses. ${ }^{11}$

\section{Phase II clinical trials}

Results from the repeated-dose Phase I pilot study in healthy postmenopausal women indicated that the appropriate ospemifene dose range was between $25 \mathrm{mg}$ /day and $100 \mathrm{mg} /$ day, as doses $<100 \mathrm{mg}$ would avoid some adverse estrogenic side effects while maintaining beneficial estrogenic effects, especially in the vaginal epithelium. Two Phase II studies of ospemifene were conducted employing the lower dose range, one of which was a placebo-controlled trial ${ }^{25-27}$ and one that employed raloxifene as an active SERM comparator. ${ }^{28,29}$ In addition to the treatment of VVA, other targeted indications evaluated during the Phase II development of ospemifene included postmenopausal osteoporosis, treatment of menopausal symptoms, and cardiovascular disease.

\section{Placebo-controlled, double-blind trial in postmenopausal women}

This first Phase II study was performed in a total of 160 healthy postmenopausal women who were randomized to receive placebo or ospemifene at $30 \mathrm{mg} /$ day, $60 \mathrm{mg} /$ day, or $90 \mathrm{mg}$ /day taken with food. ${ }^{25-27}$ A separate Phase I study conducted to assess the effect of food on the oral bioavailability of ospemifene showed that administration of the drug with food increased both maximum concentration and area under the concentration-time curve by two to three times that seen when given in the fasted state. ${ }^{30}$ In addition to standard safety laboratory assessments and adverse event monitoring, endometrial safety was thoroughly monitored by measuring endometrial thickening, histologic examination of endometrial biopsies, and Ki-67 staining to evaluate endometrial proliferation. ${ }^{26}$ Potential efficacy markers assessed in this study included serum lipids (low-density lipoprotein, highdensity lipoprotein, total cholesterol, and triglycerides) and vascular markers to evaluate cardiovascular disease treatment potential, ${ }^{27}$ serum and urinary bone markers to assess bone turnover, ${ }^{25}$ vaginal maturation index to assess the potential treatment of VVA, and the Kupperman index to assess the potential treatment of menopausal symptoms. ${ }^{26}$ The Kupperman index is a numerical index that scores eleven 
different menopausal symptoms, including hot flashes, insomnia, nervousness, vertigo, headache, and heart palpitations. Each symptom is scored on a scale from 0 to 3 , with 0 being no symptoms and 3 being the most severe.

\section{Ospemifene compared to raloxifene}

The design of this Phase II study was similar to the placebocontrolled study, except that raloxifene was included as an active comparator used in place of the placebo. ${ }^{26,29}$ At the time this study was conducted, raloxifene had already been FDA-approved for the prevention ${ }^{31}$ and treatment ${ }^{32}$ of postmenopausal osteoporosis. This 12-week, doubleblind, randomized trial included a total of 118 healthy postmenopausal women randomly assigned to treatment with either ospemifene $(30 \mathrm{mg} /$ day, $60 \mathrm{mg} /$ day, or $90 \mathrm{mg} /$ day) or raloxifene $(60 \mathrm{mg} /$ day $)$. The goals of this study were to: (1) evaluate the effects of ospemifene compared to raloxifene on hormonal status, serum lipids, genital tract, and tolerability in postmenopausal women; ${ }^{29}$ and (2) assess the effects of ospemifene on biochemical markers of bone turnover in postmenopausal women compared to raloxifene, ${ }^{28}$ which was an approved treatment and preventive agent for postmenopausal osteoporosis. Safety and efficacy evaluations in this study were similar to the placebo-controlled Phase II study.

\section{Summary of Phase II clinical trials results}

In these Phase II studies, ospemifene was again well tolerated, and routine safety assessments indicated no safety issues. ${ }^{26,29}$ Similar to observations from the Phase I repeateddose pilot study, results from the Phase II studies showed that ospemifene had significant, beneficial estrogenic effects on the vaginal maturation index. In the placebo-controlled Phase II study, ospemifene exerted a clear estrogenic effect on the vaginal epithelium at all doses as assessed by the percent change in parabasal, intermediate, and superficial cells of vaginal smears. Parabasal cells were significantly decreased, while ospemifene treatment produced significant increases in intermediate and superficial cells compared to placebo at all doses except for superficial cells at the $30 \mathrm{mg}$ dose. ${ }^{26}$ Similar results were observed in the Phase II study that compared ospemifene to raloxifene. Decreases in parabasal cells and increases in intermediate and superficial cells were seen at all doses of ospemifene, while no meaningful changes were observed with raloxifene treatment. Significant differences between the three ospemifene doses and raloxifene were demonstrated, except for parabasal cells at the $60 \mathrm{mg}$ dose. ${ }^{29}$ Overall, results from the Phase I and Phase II studies clearly demonstrated the potential benefits of ospemifene in the treatment of VVA due to menopause.

\section{Phase III clinical trials}

Based on the results obtained from the Phase I and Phase II trials, the treatment of postmenopausal VVA was selected as the target indication for the Phase III development of ospemifene. This selection was based on the fact that: (1) the clinical data were clear, compelling, and consistent with objective clinical indicators such as the vaginal maturation index; (2) the FDA had already established clear guidelines for obtaining approval for the treatment of VVA; (3) the Phase II studies showed that the dose of ospemifene needed to treat VVA would be at the low end of the tested dose range, thus minimizing potential side effects; (4) there were no existing drugs in the same class as ospemifene that had been approved for the treatment of VVA, which meant that ospemifene would not need to be compared to any existing medications in Phase III; and (5) the number of postmenopausal women suffering from the symptoms of VVA was large and underserved by existing products. ${ }^{1}$

A total of five Phase III trials of ospemifene were conducted. The first pivotal Phase III study ${ }^{33}$ evaluated the efficacy of ospemifene at doses of $30 \mathrm{mg}$ and $60 \mathrm{mg}$ in treating VVA as assessed by four co-primary endpoints: increase in superficial cells and decrease in parabasal cells of the vaginal maturation index, decrease in vaginal $\mathrm{pH}$, and improvement in the self-identified most bothersome symptom, which could include vaginal dryness, itching, dysuria, bleeding, or dyspareunia. The second pivotal Phase III trial, which was actually composed of two identically designed studies focusing on the treatment of dyspareunia ${ }^{34}$ and vaginal dryness, ${ }^{35}$ respectively, evaluated the efficacy of $60 \mathrm{mg}$ ospemifene at the same four co-primary endpoints. Long-term safety and efficacy were the focus of the last two Phase III clinical trials, one that was a 40-week extension of the first pivotal 12-week Phase III study ${ }^{36,37}$ and one that was a dedicated 52-week long-term safety study evaluating daily dosing with $60 \mathrm{mg}$ ospemifene. ${ }^{38}$

\section{First pivotal Phase III study}

This first double-blind, randomized, placebo-controlled Phase III trial of ospemifene included 826 postmenopausal patients with symptomatic VVA. ${ }^{33}$ Inclusion criteria stipulated that patients must have $<5 \%$ superficial cells on vaginal smear, a vaginal $\mathrm{pH}>5$ and at least one most bothersome symptom. Patients were randomized to treatment with either placebo or ospemifene (30 mg or $60 \mathrm{mg}$ ) given once daily for 
12 weeks. At the end of treatment, patients were given the option of entering the long-term safety extension study. In accordance with FDA guidance, all patients were provided vaginal lubricant to use as needed. The four co-primary endpoints discussed earlier were analyzed using previously agreed upon statistical methods with the FDA. Due to the inclusion of more than one dose, efficacy had to be evaluated using a step-down approach. Specifically, statistical significance had to be observed at the $60 \mathrm{mg}$ dose before the $30 \mathrm{mg}$ dose could be considered. Likewise, the multiplicity of VVA symptom evaluation was addressed with a step-down procedure, where statistically significant improvement in vaginal dryness had to be demonstrated before dyspareunia could be evaluated. Routine adverse event monitoring, standard laboratory assessments and evaluations of endometrial thickness, and histology by biopsy were performed.

The results showed that ospemifene was well tolerated, with $86 \%, 80 \%$, and $85 \%$ of patients completing the study in the placebo and the $30 \mathrm{mg}$ and $60 \mathrm{mg}$ ospemifene groups, respectively. Treatment emergent adverse events were similar in all groups. Serious adverse events were reported in nine patients (four patients in the placebo group, five patients at $30 \mathrm{mg}$ ospemifene, and none at the $60 \mathrm{mg}$ dose). The most common adverse event was hot flashes, which had an incidence of 3\% in the placebo group, $10 \%$ in the ospemifene $30 \mathrm{mg}$ group, and $8 \%$ in the ospemifene $60 \mathrm{mg}$ group. Few patients experienced severe hot flashes or discontinued because of hot flashes, but the $30 \mathrm{mg}$ dose appeared slightly worse than either the placebo or the $60 \mathrm{mg}$ dose of ospemifene. Urinary tract infections were slightly more prevalent in the ospemifene-treated groups, which may have been related to a higher frequency of sexual activity. Endometrial thickness differed by $<1 \mathrm{~mm}$ between the three treatment groups at the end of the 12-week treatment period, and no cases of endometrial hyperplasia were observed in any group. The efficacy of ospemifene was clearly demonstrated by all objective measures. Compared to placebo $(+2 \%)$, the percentage of superficial cells was significantly increased by ospemifene treatment at both the $30 \mathrm{mg}(+8 \%)$ and $60 \mathrm{mg}$ doses $(+11 \%)$. Parabasal cells were significantly decreased at both $30 \mathrm{mg}(-22 \%)$ and $60 \mathrm{mg}(-30 \%)$ ospemifene compared to a $4 \%$ increase in the placebo group. Ospemifene treatment also resulted in significant decreases in vaginal $\mathrm{pH}$, with the $30 \mathrm{mg}$ and $60 \mathrm{mg}$ doses decreasing $\mathrm{pH}$ by 0.7 and 1.0 points, respectively, compared to a decrease of 0.1 point in placebotreated patients. ${ }^{33}$

The two most bothersome symptoms that occurred frequently enough for analysis were dyspareunia (46\%) and vaginal dryness $(39 \%)$. Ospemifene treatment resulted in significant improvement in these symptoms, particularly at the $60 \mathrm{mg}$ dose. After 12 weeks of treatment, the vaginal dryness symptom score decreased significantly by 1.22 and 1.26 points in the $30 \mathrm{mg}$ and $60 \mathrm{mg}$ groups, respectively, compared to a decrease of 0.84 points in the placebo group. Likewise, ospemifene treatment decreased dyspareunia symptom scores by 1.19 and 1.02 points at the $60 \mathrm{mg}$ and $30 \mathrm{mg}$ doses, respectively, compared to a 0.89 -point decrease in the placebo group. Though the decrease at both ospemifene doses exceeded that in the placebo group, the difference reached significance only at the $60 \mathrm{mg}$ dose. Each symptom was assessed on a four-point scale specified as none (0), mild (1), moderate (2), or severe (3). Approximately $30 \%$ of women used lubricant at the beginning of the study. Lubricant use decreased in all groups as the study progressed, with the decrease being greater in the active treatment groups. ${ }^{33}$

\section{Second pivotal Phase III trial (dyspareunia)}

Based on the results of the first pivotal Phase III study as well as the Phase I and Phase II studies showing inferiority of the $30 \mathrm{mg}$ dose, the second pivotal Phase III study (both the dyspareunia and vaginal dryness substudies) compared $60 \mathrm{mg}$ ospemifene to placebo. ${ }^{34,35}$ As in the first pivotal Phase III study, lubricants were provided to all study participants. Since the only VVA symptoms that occurred frequently enough to be effectively studied were vaginal dryness and dyspareunia, these symptoms were analyzed in separate studies. The Female Sexual Satisfaction Survey, a standardized scale used to evaluate quality of life improvements, was also included. Other than these modifications, the two additional Phase III studies, one in dyspareunia ${ }^{34}$ and one in vaginal dryness, ${ }^{35}$ were identical to the first pivotal Phase III trial. These 12-week studies were conducted in parallel and evaluated the safety and efficacy of ospemifene $60 \mathrm{mg}$ compared to placebo using the same measures as in the first pivotal Phase III study.

The dyspareunia study randomized 605 postmenopausal patients with a diagnosis of VVA who self-identified a most bothersome symptom of dyspareunia for treatment with either placebo or ospemifene $60 \mathrm{mg} .{ }^{34}$ Efficacy was again clearly demonstrated by significant improvement in all four co-primary endpoints. The decrease in the percentage of parabasal cells, the increase in the percentage of superficial cells, the decrease in vaginal $\mathrm{pH}$, and the decrease in the severity of dyspareunia symptoms were similar to previous studies and were highly significantly different compared to placebo. As in the first Phase III study, lubricant use was 
similar in the two groups early in the study and decreased during treatment in both groups, more so with ospemifene treatment than placebo. The drug was again well tolerated, with more ospemifene-treated patients (92\%) completing the study compared to placebo-treated patients ( $88 \%)$, but a slightly higher percentage of ospemifene-treated patients $(4.6 \%)$ than placebo-treated patients $(3.3 \%)$ discontinuing because of adverse events. There were no treatment-related serious adverse events reported in either group. The most frequent adverse events in the ospemifene group were hot flashes $(6.6 \%$ vs $4.3 \%$ in placebo) and urinary tract infections (5.6\% vs $3.6 \%$ in placebo). Endometrial thickness increased slightly more with ospemifene treatment $(0.4 \mathrm{~mm})$ compared to placebo $(0.1 \mathrm{~mm})$, but no cases of hyperplasia were observed. ${ }^{34}$

\section{Second pivotal Phase III trial (vaginal dryness)}

The vaginal dryness study randomized 314 postmenopausal patients with a diagnosis of VVA and a self-identified most bothersome symptom of moderate-to-severe vaginal dryness to treatment with either $60 \mathrm{mg}$ ospemifene or placebo daily for 12 weeks. $^{35}$ All three objective measures of efficacy were significantly improved with ospemifene treatment. The decrease in the percentage of parabasal cells, the increase in the percentage of superficial cells, and the decrease in vaginal $\mathrm{pH}$ were similar to previous studies and were highly significant compared to placebo. Improvement in vaginal dryness was greater in the ospemifene-treated group $(-1.3)$ than the placebo-treated group $(-1.1)$, but the intent-to-treat analysis with the last visit carried forward for missing data, which is required for regulatory approval, fell short of statistical significance. The more clinically relevant per protocol analysis, which included only patients who had completed 10 weeks of treatment with $85 \%$ compliance, showed significantly greater improvement with ospemifene $(-1.4)$ compared to placebo $(-1.1)$. There were also greater proportions of patients describing either no or mild vaginal dryness $(30 \%$ vs $23 \%$ ) and patients improving by two to three levels in the symptom score (50\% vs $34 \%$ ) in the ospemifene-treated group compared to placebo-treated group. The safety profile in this study was similar to that previously reported. Hot flashes and urinary tract infections were the most frequent adverse events. Serious adverse events were similar in the two groups, but there was one case of deep vein thrombosis in the ospemifene group that was considered possibly related to treatment. Endometrial thickening was more evident in the ospemifene group than the placebo group, but no hyperplasia was reported. ${ }^{35}$

\section{Phase III long-term safety}

For endometrial safety, FDA guidance required that 100-200 patients be treated at the intended dose for 1 year, with endometrial biopsies confirming $<1 \%$ hyperplasia with $95 \%$ confidence. Some long-term safety data with endometrial biopsies were obtained from the long-term safety extension of the first pivotal Phase III trial, ${ }^{36,37}$ but in order to reach the required 1-year exposure, a dedicated Phase III long-term safety study was initiated. ${ }^{38}$ This study randomized postmenopausal women with a diagnosis of VVA and an intact uterus to treatment with $60 \mathrm{mg}$ ospemifene or placebo daily for 12 months using a 6:1 randomization schedule. Safety assessments included endometrial biopsy, endometrial thickness, and breast and gynecological exams. Efficacy at 12 weeks was also examined with vaginal maturation index and vaginal $\mathrm{pH}$, but unlike previous studies there was no assessment of symptomatic efficacy. Out of the 426 patients randomized, 363 patients received ospemifene and 63 patients received placebo. A total of 349 patients completed the study: 294 patients in the ospemifene group and 55 patients in the placebo group. After 12 weeks of treatment, changes in the objective measures of efficacy including the decrease in parabasal cells, increase in superficial cells, and decrease in vaginal $\mathrm{pH}$ were similar in magnitude to those previously observed and were highly significant. ${ }^{38}$ Visual evaluation of the vagina at 52 weeks of treatment showed significant improvement in petechiae, pallor, friability, vaginal dryness, and vaginal redness in patients treated with $60 \mathrm{mg}$ ospemifene compared to placebo. ${ }^{38}$ Safety outcomes are presented in the "Comprehensive clinical safety summary" section.

\section{Phase IIIVVA efficacy summary}

A summary of ospemifene's efficacy as assessed by vaginal maturation index, vaginal $\mathrm{pH}$, and VVA symptom relief from the Phase III trials is presented in Tables 1 and 2. The objective measures were all consistently positive and highly statistically significant in all four Phase III studies in which they were assessed. ${ }^{33-35,38}$ Although changes in the vaginal maturation index and vaginal $\mathrm{pH}$ were significant at the 30 mg dose, the magnitude was less than that observed at 60 mg. Parabasal cells decreased $30 \%-40 \%$ at the $60 \mathrm{mg}$ dose, with small and inconsistent changes in the placebo group (Table 1). Superficial cells increased 5\%-10\% with little if any change in the placebo group. Vaginal $\mathrm{pH}$ decreased by about one point at the $60 \mathrm{mg}$ dose (Table 2). As assessed by these measures, efficacy continued through 1 year of treatment. $^{38}$ 
Table I Phase III efficacy of ospemifene: vaginal maturation index

\begin{tabular}{|c|c|c|c|c|c|c|c|c|c|}
\hline \multirow[t]{2}{*}{ Study } & \multicolumn{3}{|c|}{ Parabasal cells $(\% \Delta)^{a}$} & \multicolumn{3}{|c|}{ Superficial cells $(\% \Delta)^{a}$} & \multicolumn{3}{|c|}{ Number of patients (n) } \\
\hline & Placebo & $30 \mathrm{mg}$ & $60 \mathrm{mg}$ & Placebo & $30 \mathrm{mg}$ & $60 \mathrm{mg}$ & Placebo & $30 \mathrm{mg}$ & $60 \mathrm{mg}$ \\
\hline First pivotal phase III $^{33}$ & 4.0 & $-21.9^{b}$ & $-30.1^{b}$ & 2.2 & $7.8^{\mathrm{b}}$ & $10.8^{\mathrm{b}}$ & 268 & 282 & 276 \\
\hline Phase III dyspareunia (ITT) $)^{34}$ & 0.0 & - & $-40.2^{c}$ & 1.7 & - & $12.3^{c}$ & 302 & - & 303 \\
\hline Phase III dyspareunia (PP) ${ }^{34}$ & -0.2 & - & $-42.1^{c}$ & 1.9 & - & $13.2^{\mathrm{c}}$ & 251 & - & 255 \\
\hline Phase III vaginal dryness (ITT) ${ }^{35}$ & -3.9 & - & $-31.7^{b}$ & 0.0 & - & $7.0^{\mathrm{b}}$ & 154 & - & 160 \\
\hline Phase III vaginal dryness (PP) $)^{35}$ & -4.7 & - & $-36.6^{b}$ & 0.0 & - & $8.0^{\mathrm{b}}$ & 137 & - & 127 \\
\hline Phase III long-term safety ${ }^{38}$ & 0 & - & $-40^{c}$ & 0 & - & $5^{c}$ & 63 & - & 363 \\
\hline
\end{tabular}

Notes: aBaseline to week 12. ${ }^{b} P<0.001$ compared to placebo. ${ }^{c} P<0.0001$ compared to placebo.

Abbreviations: ITT, intent to treat; PP, per protocol.

With respect to symptomatic relief of dyspareunia and vaginal dryness as measured on the four-point scale, the $60 \mathrm{mg}$ dose proved to be superior compared to placebo and $30 \mathrm{mg}$ ospemifene. For dyspareunia, symptomatic relief was significant at the $60 \mathrm{mg}$ dose in both the pivotal Phase III studies. ${ }^{33,34}$ The $30 \mathrm{mg}$ dose was better than placebo, but the change failed to reach statistical significance (Table 2). The number of patients who experienced an improvement in symptom score of at least two points was higher with $60 \mathrm{mg}$ ospemifene than either placebo or $30 \mathrm{mg}$ ospemifene. ${ }^{33}$ Symptomatic improvement in patients with vaginal dryness was significantly better at both $30 \mathrm{mg}$ and $60 \mathrm{mg}$ ospemifene in the first pivotal Phase III trial (Table 2). ${ }^{33}$ This was true when measured by either the average on the four-point symptom scale or the number of patients experiencing improvement of at least two points on the scale. In the patients included in the Phase III vaginal dryness trial, the magnitude of symptom improvement was similar to the dyspareunia study, but by intent-to-treat analysis, it fell slightly short of statistical significance. In the more clinically relevant per protocol analysis, the difference was significant (Table 2). ${ }^{35}$ Other secondary analyses of efficacy in this group such as number of responders, number of patients showing at least two levels of improvement, and the number of patients experiencing complete symptom relief all supported the conclusion that vaginal dryness was indeed effectively treated in this study. ${ }^{35}$

\section{Comprehensive clinical safety summary}

Unlike efficacy, which is best evaluated by considering the results of individual studies that are designed and statistically powered to show clinical effects, drug safety is better assessed as a reflection of the total exposures from all studies combined. A total of 1,892 patients were exposed to ospemifene during the Phase II and Phase III studies, with an average duration of exposure of 182 days. This represents approximately 1,000 patient years of exposure under monitored conditions, the majority of which occurred at the $60 \mathrm{mg}$ dose. A patient year is the exposure of one patient to the study drug for 1 year at the prescribed dosing schedule. More than 400 patients exceeded 1 year of treatment with ospemifene. All patients included in the ospemifene Phase II and Phase III clinical trials were postmenopausal with an average age of 59 years, and $86 \%$ were on concomitant medications.

In the clinical studies, no clinically significant changes in routine safety assessments, including hematology, chemistry, and urinalysis, were observed. The most common treatment emergent adverse events at the $60 \mathrm{mg}$ dose were hot flashes ( $7.5 \%$ vs $2.6 \%$ placebo), vaginal discharge (3.8\% vs $0.3 \%$ placebo), muscle spasms ( $3.2 \%$ vs $0.9 \%$ placebo)

Table 2 Phase III efficacy of ospemifene: vaginal $\mathrm{pH}$ and symptoms of vulvar and vaginal atrophy

\begin{tabular}{|c|c|c|c|c|c|c|c|c|c|}
\hline \multirow[t]{2}{*}{ Study } & \multicolumn{3}{|c|}{ Vaginal pH $(\Delta)^{\mathrm{a}}$} & \multicolumn{3}{|c|}{ Most bothersome symptom $(\Delta)^{\mathrm{a}}$} & \multicolumn{3}{|c|}{ Number of patients $(n)$} \\
\hline & Placebo & $30 \mathrm{mg}$ & $60 \mathrm{mg}$ & Placebo & $30 \mathrm{mg}$ & $60 \mathrm{mg}$ & Placebo & $30 \mathrm{mg}$ & $60 \mathrm{mg}$ \\
\hline \multirow[t]{2}{*}{ First pivotal phase $\mathrm{III}^{33}$} & -0.10 & $-0.67^{b}$ & $-1.0 I^{b}$ & $-0.84 \%$ & $-1.22^{\mathrm{b}, \mathrm{c}}$ & $-1.26^{\mathrm{b}, \mathrm{c}} /$ & 268 & 282 & 276 \\
\hline & & & & $-0.89^{d}$ & $-1.02^{\mathrm{d}}$ & $-1.19^{\mathrm{d}, \mathrm{e}}$ & & & \\
\hline Phase III dyspareunia (ITT) ${ }^{34}$ & -0.07 & - & $-0.94^{f}$ & -1.2 & - & $-1.5^{f}$ & 302 & - & 303 \\
\hline Phase III dyspareunia (PP) ${ }^{34}$ & -0.08 & - & $-1 . I^{f}$ & -1.2 & - & $-1.6^{\mathrm{g}}$ & 251 & - & 255 \\
\hline Phase III vaginal dryness (ITT) ${ }^{35}$ & -0.25 & - & $-0.95^{b}$ & -1.1 & - & -1.3 & 154 & - & 160 \\
\hline Phase III vaginal dryness $(\mathrm{PP})^{35}$ & -0.23 & - & $-0.99^{b}$ & -1.1 & - & $-1.4^{\mathrm{h}}$ & 137 & - & 127 \\
\hline
\end{tabular}

Notes: ${ }^{a}$ From baseline to 12 weeks. ${ }^{b} P<0.001$ compared to placebo. ${ }^{\mathrm{C} V a g i n a l}$ dryness. ${ }^{\mathrm{d}} \mathrm{Dys}$ pareunia. ${ }^{\mathrm{e} P}=0.023$ compared to $p$ lacebo. ${ }^{\mathrm{f}} \mathrm{P}<0.000 \mathrm{I}$ compared to placebo. ${ }^{g} P=0.0004$ compared to placebo. ${ }^{h} P=0.014$ compared to placebo.

Abbreviations: ITT, intent to treat; PP, per protocol. 
and headache $(2.4 \%$ incidence with both ospemifene and placebo). ${ }^{39}$ Although vasomotor symptoms were more common in ospemifene-treated patients, the symptoms were well tolerated and rarely led to study discontinuation. ${ }^{38}$ Urinary tract infections were also more common in ospemifenetreated patients, particularly early in the trials. It has been speculated that this may be related to more frequent sexual activity, implying that the treatment was working, but this has not been confirmed. ${ }^{33}$ Urinary tract infections seemed to occur at similar rates in the ospemifene and placebo groups as treatment continued. ${ }^{37}$ All serious treatment emergent adverse events not necessarily related to drug treatment were slightly higher in patients treated with $60 \mathrm{mg}$ ospemifene compared to placebo when evaluated by patient, but lower for $60 \mathrm{mg}$ ospemifene than in the placebo patients when evaluated by exposure. ${ }^{36-38}$ No deaths occurred in any of the ospemifene clinical trials.

Of particular interest with SERMs is the frequency of cardiovascular events. Six ospemifene-treated patients $(0.3 \%)$ and one placebo-treated patient $(0.1 \%)$ discontinued due to a cardiovascular event. For patients treated with ospemifene, these events included two deep vein thromboses, three cerebrovascular accidents (strokes), and one myocardial infarction that occurred in a patient who had pre-existing cardiovascular disease and a long history of type 2 diabetes mellitus and hypertension. ${ }^{35,36,38}$ Of the three strokes in ospemifene-treated patients, one was not considered treatment related. ${ }^{38}$ There were no cases of thromboembolism reported. Two cardiovascular events, a stroke, and a deep vein thrombosis, occurred in the placebo-treated patients. ${ }^{34,39}$ The rate of occurrence of any cardiovascular event was approximately six per 1,000 patient years of treatment. The incidence of deep vein thrombosis was approximately $0.1 \%$ in ospemifene-treated patients, which is lower than that reported for recently approved SERMs, and was the same as that observed in the placebo-treated patients. ${ }^{39}$ The incidence of stroke in both ospemifene-treated and placebo-treated patients was also approximately $0.1 \% .^{38,39}$ No electrocardiogram changes were observed in patients taking ospemifene. A detailed clinical evaluation of the impact of ospemifene treatment, as required by the FDA, was negative. ${ }^{2}$

Endometrial safety was of course a major point of emphasis during the clinical development of ospemifene. Adverse estrogenic effects in the uterus have proven to be a major stumbling block in the development of new SERMs. ${ }^{40}$ Transvaginal ultrasound before, during, and after treatment was used to monitor and detect changes in the endometrium. Compared to placebo, which showed no change, mean endometrial thickness increased by less than $1 \mathrm{~mm}$ during 12 months of treatment with $60 \mathrm{mg}$ ospemifene. ${ }^{38}$ The number of patients with endometrial thickening greater than $8 \mathrm{~mm}$ at the end of 12 months of treatment was higher in ospemifene-treated patients $(1.0 \%)$ compared to placebotreated patients $(0 \%) .{ }^{37}$ No cases of endometrial hyperplasia or carcinoma were detected by biopsy at the end of 12 months of treatment. ${ }^{37}$ Although one patient did have a biopsy that showed endometrial hyperplasia without atypia 3 months after the last dose of ospemifene, ${ }^{38}$ one case in 347 biopsies after 12 months of treatment is well below the threshold for concern. Five patients who received $60 \mathrm{mg}$ of ospemifene had an endometrial polyp on biopsy (1.4\%), only one of which $(0.3 \%)$ was confirmed to be a true polyp. ${ }^{38}$ Bleeding or spotting occurred in $1.4 \%$ of patients with an intact uterus treated with ospemifene ${ }^{38}$ compared to $0.7 \%$ of similar placebo-treated patients. ${ }^{34}$

In postmenopausal women taking estrogen-based products for the treatment of menopausal symptoms, breast safety has become a key concern following the results of the Women's Health Initiative trial of estrogen plus progestin. ${ }^{5}$ After 1 year of treatment, ospemifene demonstrated a very good breast safety profile. While there were reports of breast tenderness, breast mass, and breast pain, the frequency was low, and similar to that which occurred in the placebo group. ${ }^{37,38}$ There were two cases of breast cancer reported in 300 patient years of placebo treatment, which is a frequency that is to be expected in this population. Interestingly, in the 805 patient years of treatment with ospemifene, no cases of breast cancer were reported, ${ }^{36-38}$ which is consistent with extensive preclinical data in rodent models of breast cancer showing that ospemifene is effective as a breast cancer chemopreventive agent, similar to tamoxifen..$^{23,41-44}$

\section{Discussion}

Ospemifene, currently being sold under the trade name Osphena, is an SERM that was approved by the FDA in early 2013 for the treatment of moderate-to-severe dyspareunia associated with VVA due to menopause. As the first nonhormonal, non-estrogen agent approved for the treatment of this condition, ospemifene represents a significant milestone in postmenopausal women's health, helping satisfy an unmet medical need for a non-hormonal alternative to estrogenbased products, the use of which has been associated with increased risks of breast cancer, stroke, and cardiovascular disease, ${ }^{3,5,8,45,46}$ for the treatment of VVA symptoms. Among the different SERMs that have received FDA approval, which include tamoxifen, toremifene, raloxifene, and bazedoxifene 
(approved as a single agent only in the Europe), ospemifene is unique because it exerts a nearly full estrogen agonist effect in the vagina, ${ }^{20,21}$ making it an effective treatment for VVA symptoms, ${ }^{33-35,38}$ while tamoxifen may aggravate this condition. ${ }^{47}$ Although the FDA did not grant approval for the treatment of vaginal dryness, the close association of the symptoms of vaginal dryness and dyspareunia to VVA as documented by objective criteria and multiple parameter analyses that support efficacy in the treatment of vaginal dryness, there is little doubt that ospemifene is an effective treatment for vaginal dryness associated with VVA due to menopause. ${ }^{33,35}$

Data from multiple Phase III clinical trials have clearly demonstrated the efficacy of ospemifene in treating VVA as assessed by significant improvement in the vaginal maturation index (increases in superficial cells and decreases in parabasal cells), significant decreases in vaginal $\mathrm{pH}$, and significant improvement in the self-identified most bothersome symptoms of dyspareunia and vaginal dryness. ${ }^{33-38}$ Based on the results of Phase III long-term safety studies showing that ospemifene has no clinically relevant estrogenic effects in the endometrium and a very low incidence of cardiovascular events, similar to placebo, following daily treatment with $60 \mathrm{mg}$ for 1 year, ospemifene is a safe alternative to estrogen-based products for the treatment of VVA due to menopause. ${ }^{37,38}$ Although vasomotor symptoms were more common in ospemifene-treated patients - hot flashes were the most frequently encountered adverse event - they were well tolerated and rarely led to study discontinuation. ${ }^{38}$ With regard to breast-related issues, ospemifene demonstrated a good safety profile. While some cases of breast tenderness and pain were observed, the incidence was low and not significantly different from placebo. ${ }^{37,38}$ Most importantly, no cases of breast cancer were seen in the ospemifene-treated patients. ${ }^{36-38}$

Interestingly, at the time ospemifene was approved, the FDA required that its labeling include a boxed warning similar to estrogen-based products, a warning considered unwarranted by some, ${ }^{48}$ as well as a warning against its use in women who have or may develop breast cancer. ${ }^{39}$ While it is true that ospemifene does exert a slight estrogenic effect in the endometrium, published preclinical and clinical data show that the magnitude of this effect is far less than that of steroidal estrogen and was found to be clinically insignificant. $^{20,21,23,49-52}$ Furthermore, following 52 weeks of daily treatment with $60 \mathrm{mg}$, the effects of ospemifene in the endometrium, based on histology and ultrasonography results, appeared to be more similar to the SERM raloxifene than to estrogen. ${ }^{49} \mathrm{With}$ regard to the breast cancer warning, it is true that the currently available clinical data are insufficient to make any claim that ospemifene has beneficial effects in either the treatment or prevention of breast cancer; however, there are no clinical data showing that ospemifene would increase the risk of breast cancer either. In fact, virtually all data generated to date suggest that ospemifene, similar to other FDA-approved SERMs, acts as an antiestrogen in breast tissue and is more likely to have beneficial than detrimental effects. ${ }^{44}$ Further clinical study is needed to establish the breast safety of ospemifene and to assess its potential as a breast cancer chemopreventive agent.

\section{Conclusion}

Ospemifene is an SERM that was approved by FDA in 2013 for the treatment of moderate-to-severe dyspareunia associated with VVA due to menopause, making it the first non-hormonal agent to receive such approval. The results of several Phase III clinical trials have clearly demonstrated that ospemifene is highly effective in improving the vaginal maturation index, decreasing vaginal $\mathrm{pH}$, and treating the most bothersome symptoms of dyspareunia and vaginal dryness. Ospemifene possesses a nearly full estrogen agonist effect in the vaginal epithelium, making it uniquely suited among the currently approved SERMs for the treatment of VVA in postmenopausal women. Long-term safety studies have established that while ospemifene does exert a slight estrogenic effect in the endometrium, this effect is clinically insignificant and similar to that of the SERM raloxifene. Hot flashes were the most common side effect seen in clinical trials, but they were well tolerated and mostly mild to moderate in severity. Ospemifene demonstrated a good breast safety profile, and the incidence of cardiovascular events was similar to placebo. Thus, ospemifene appears to be a safe and effective alternative to estrogen-based products for the treatment of VVA-related symptoms.

\section{Acknowledgment}

The authors would like to thank Timothy Cadman and Dr Lin Soe for their critical review of the manuscript.

\section{Disclosure}

Drs Gregory T Wurz and Chiao-Jung Kao have no conflicts of interest to declare. Dr Michael DeGregorio is one of the original inventors of ospemifene and has a potential conflict of interest. 


\section{References}

1. DeGregorio MW, Cadman TB, Wurz GT. Painful Sex Associated with Menopause: Interpreting FDA Warnings When Choosing a Treatment for Dyspareunia. New York: Nova Science Publishers, Inc; 2014.

2. DeGregorio MW, Zerbe RL, Wurz GT. Ospemifene: a first-in-class, non-hormonal selective estrogen receptor modulator approved for the treatment of dyspareunia associated with vulvar and vaginal atrophy. Steroids. 2014;90:82-93.

3. Chlebowski RT, Anderson GL, Gass M, et al. Estrogen plus progestin and breast cancer incidence and mortality in postmenopausal women. JAMA. 2010;304(15):1684-1692.

4. Nelson HD, Walker M, Zakher B, Mitchell J. Menopausal hormone therapy for the primary prevention of chronic conditions: a systematic review to update the US. Preventive Services Task Force recommendations. Ann Intern Med. 2012;157(2):104-113.

5. Rossouw JE, Anderson GL, Prentice RL, et al. Risks and benefits of estrogen plus progestin in healthy postmenopausal women: principal results from the Women's Health Initiative randomized controlled trial. JAMA. 2002;288(3):321-333.

6. Rozenberg S, Vandromme J, Antoine C. Postmenopausal hormone therapy: risks and benefits. Nat Rev Endocrinol. 2013;9(4):216-227.

7. Tolonen A, Koskimies P, Turpeinen M, Uusitalo J, Lammintausta R, Pelkonen O. Ospemifene metabolism in humans in vitro and in vivo: metabolite identification, quantitation, and CYP assignment of major hydroxylations. Drug Metabol Drug Interact. 2013;28(3):153-161.

8. Anderson GL, Limacher M, Assaf AR, et al. Effects of conjugated equine estrogen in postmenopausal women with hysterectomy: the Women's Health Initiative randomized controlled trial. JAMA. 2004;291(14):1701-1712.

9. NAMS. Management of symptomatic vulvovaginal atrophy: 2013 position statement of the North American Menopause Society. Menopause. 2013;20(9):888-902.

10. Burich R, DeGregorio M. Current treatment options for vulvovaginal atrophy. Expert Rev Obstet Gynecol. 2011;6(2):141-151.

11. Voipio SK, Komi J, Kangas L, Halonen K, DeGregorio MW, Erkkola RU. Effects of ospemifene (FC-1271a) on uterine endometrium, vaginal maturation index, and hormonal status in healthy postmenopausal women. Maturitas. 2002;43(3):207-214.

12. Calleja-Agius J, Brincat MP. Urogenital atrophy. Climacteric. 2009;12(4): 279-285.

13. Mac Bride MB, Rhodes DJ, Shuster LT. Vulvovaginal atrophy. Mayo Clin Proc. 2010;85(1):87-94.

14. Lewis V. Undertreatment of menopausal symptoms and novel options for comprehensive management. Curr Med Res Opin. 2009;25(11): 2689-2698.

15. Nappi RE, Palacios S. Impact of vulvovaginal atrophy on sexual health and quality of life at postmenopause. Climacteric. 2014;17(1):3-9.

16. Santoro N, Komi J. Prevalence and impact of vaginal symptoms among postmenopausal women. J Sex Med. 2009;6(8):2133-2142.

17. Al-Baghdadi O, Ewies AA. Topical estrogen therapy in the management of postmenopausal vaginal atrophy: an up-to-date overview. Climacteric. 2009;12(2):91-105.

18. Lara LA, Useche B, Ferriani RA, et al. The effects of hypoestrogenism on the vaginal wall: interference with the normal sexual response. $J$ Sex Med. 2009;6(1):30-39.

19. Goldstein I, Alexander JL. Practical aspects in the management of vaginal atrophy and sexual dysfunction in perimenopausal and postmenopausal women. $J$ Sex Med. 2005;2(suppl 3):154-165.

20. Kangas L, Unkila M. Tissue selectivity of ospemifene: pharmacologic profile and clinical implications. Steroids. 2013;78(12-13):1273-1280.

21. Unkila M, Kari S, Yatkin E, Lammintausta R. Vaginal effects of ospemifene in the ovariectomized rat preclinical model of menopause. $J$ Steroid Biochem Mol Biol. 2013;138:107-115.

22. Nijland EA, Weijmar Schultz WC, Davis SR. Effects of tibolone and raloxifene on health-related quality of life and sexual function. Maturitas. 2007;58(2):164-173.
23. Qu Q, Zheng H, Dahllund J, et al. Selective estrogenic effects of a novel triphenylethylene compound, FC1271a, on bone, cholesterol level, and reproductive tissues in intact and ovariectomized rats. Endocrinology. 2000;141(2):809-820.

24. DeGregorio MW, Wurz GT, Taras TL, Erkkola RU, Halonen KH, Huupponen RK. Pharmacokinetics of (deaminohydroxy) toremifene in humans: a new, selective estrogen-receptor modulator. Eur J Clin Pharmacol. 2000;56(6-7):469-475.

25. Komi J, Heikkinen J, Rutanen EM, Halonen K, Lammintausta R, Ylikorkala O. Effects of ospemifene, a novel SERM, on biochemical markers of bone turnover in healthy postmenopausal women. Gynecol Endocrinol. 2004;18(3):152-158.

26. Rutanen EM, Heikkinen J, Halonen K, Komi J, Lammintausta R, Ylikorkala O. Effects of ospemifene, a novel SERM, on hormones, genital tract, climacteric symptoms, and quality of life in postmenopausal women: a double-blind, randomized trial. Menopause. 2003;10(5): 433-439.

27. Ylikorkala O, Cacciatore B, Halonen K, et al. Effects of ospemifene, a novel SERM, on vascular markers and function in healthy, postmenopausal women. Menopause. 2003;10(5):440-447.

28. Komi J, Lankinen KS, DeGregorio M, et al. Effects of ospemifene and raloxifene on biochemical markers of bone turnover in postmenopausal women. J Bone Miner Metab. 2006;24(4):314-318.

29. Komi J, Lankinen KS, Härkönen P, et al. Effects of ospemifene and raloxifene on hormonal status, lipids, genital tract, and tolerability in postmenopausal women. Menopause. 2005;12(2): 202-209.

30. Koskimies P, Katila K, Lammintausta R, et al. Oral bioavailability of ospemifene improves with food intake. Int J Clin Pharmacol Ther. 2013; 51(10): 787-794

31. Delmas PD, Bjarnason NH, Mitlak BH, et al. Effects of raloxifene on bone mineral density, serum cholesterol concentrations, and uterine endometrium in postmenopausal women. $N$ Engl J Med. 1997; 337(23):1641-1647.

32. Ettinger B, Black DM, Mitlak BH, et al. Reduction of vertebral fracture risk in postmenopausal women with osteoporosis treated with raloxifene: results from a 3-year randomized clinical trial. Multiple Outcomes of Raloxifene Evaluation (MORE) Investigators. JAMA. 1999;282(7):637-645.

33. Bachmann GA, Komi JO. Ospemifene effectively treats vulvovaginal atrophy in postmenopausal women: results from a pivotal phase 3 study. Menopause. 2010;17(3):480-486.

34. Portman DJ, Bachmann GA, Simon JA. Ospemifene, a novel selective estrogen receptor modulator for treating dyspareunia associated with postmenopausal vulvar and vaginal atrophy. Menopause. 2013; 20(6):623-630.

35. Portman D, Palacios S, Nappi RE, Mueck AO. Ospemifene, a nonoestrogen selective oestrogen receptor modulator for the treatment of vaginal dryness associated with postmenopausal vulvar and vaginal atrophy: a randomised, placebo-controlled, phase III trial. Maturitas. 2014;78(2):91-98

36. Simon J, Portman D, Mabey RG Jr. Long-term safety of ospemifene (52-week extension) in the treatment of vulvar and vaginal atrophy in hysterectomized postmenopausal women. Maturitas. 2014;77(3): 274-281.

37. Simon JA, Lin VH, Radovich C, Bachmann GA. One-year long-term safety extension study of ospemifene for the treatment of vulvar and vaginal atrophy in postmenopausal women with a uterus. Menopause. 2013;20(4):418-427.

38. Goldstein SR, Bachmann GA, Koninckx PR, Lin VH, Portman DJ, Ylikorkala O. Ospemifene 12-month safety and efficacy in postmenopausal women with vulvar and vaginal atrophy. Climacteric. 2014;17(2):173-182.

39. Osphena ${ }^{\mathrm{TM}}$ (Ospemifene) Prescribing Information. Florham Park, NJ: Shionogi Inc.; 2013. Available from: http://www.shionogi.com/pdf/PI/ Osphena-PI.pdf. Accessed August 29, 2014. 
40. Pinkerton JV, Goldstein SR. Endometrial safety: a key hurdle for selective estrogen receptor modulators in development. Menopause. 2010; 17(3):642-653.

41. Burich RA, Mehta NR, Wurz GT, et al. Ospemifene and 4-hydroxyospemifene effectively prevent and treat breast cancer in the MTag.Tg transgenic mouse model. Menopause. 2012;19(1):96-103.

42. Namba R, Young LJ, Maglione JE, et al. Selective estrogen receptor modulators inhibit growth and progression of premalignant lesions in a mouse model of ductal carcinoma in situ. Breast Cancer Res. 2005; 7(6):R881-R889.

43. Wurz GT, Read KC, Marchisano-Karpman C, et al. Ospemifene inhibits the growth of dimethylbenzanthracene-induced mammary tumors in Sencar mice. J Steroid Biochem Mol Biol. 2005;97(3):230-240.

44. Wurz GT, Soe LH, Degregorio MW. Ospemifene, vulvovaginal atrophy, and breast cancer. Maturitas. 2013;74(3):220-225

45. Manson JE, Chlebowski RT, Stefanick ML, et al. Menopausal hormone therapy and health outcomes during the intervention and extended poststopping phases of the Women's Health Initiative randomized trials. JAMA. 2013;310(13):1353-1368.

46. Manson JE, Hsia J, Johnson KC, et al. Estrogen plus progestin and the risk of coronary heart disease. $N$ Engl J Med. 2003;349(6):523-534.
47. Lorizio $\mathrm{W}, \mathrm{Wu} \mathrm{AH}$, Beattie MS, et al. Clinical and biomarker predictors of side effects from tamoxifen. Breast Cancer Res Treat. 2012;132(3): 1107-1118.

48. Goldstein SR. Postmenopausal dyspareunia: has the Food and Drug Administration really helped? Menopause. 2013;20(6):596-597.

49. Constantine GD, Goldstein SR, Archer DF. Endometrial safety of ospemifene: results of the phase $2 / 3$ clinical development program. Menopause. Epub 2014 June 23.

50. Kangas L, Harkonen P, Vaananen K, Peng Z. Effects of the selective estrogen receptor modulator ospemifene on bone in rats. Horm Metab Res. 2014;46(1):27-35.

51. Wurz GT, Hellmann-Blumberg U, DeGregorio MW. Pharmacologic effects of ospemifene in rhesus macaques: a pilot study. Basic Clin Pharmacol Toxicol. 2008;102(6):552-558.

52. Zheng H, Kangas L, Harkonen PL. Comparative study of the short-term effects of a novel selective estrogen receptor modulator, ospemifene, and raloxifene and tamoxifen on rat uterus. J Steroid Biochem Mol Biol. 2004;88(2):143-156.
Clinical Interventions in Aging

\section{Publish your work in this journal}

Clinical Interventions in Aging is an international, peer-reviewed journal focusing on evidence-based reports on the value or lack thereof of treatments intended to prevent or delay the onset of maladaptive correlates of aging in human beings. This journal is indexed on PubMed Central, MedLine,

\section{Dovepress}

CAS, Scopus and the Elsevier Bibliographic databases. The manuscript management system is completely online and includes a very quick and fair peer-review system, which is all easy to use. Visit http://www.dovepress. com/testimonials.php to read real quotes from published authors. 